Article

\title{
Time to cure and predictors of recovery among children aged 6-59 months with severe acute malnutrition admitted in Jimma university medical center, Southwest Ethiopia
}

\author{
Robel kabthymer ${ }^{1}{ }^{*}$, Getu Gizaw ${ }^{2}$ and Tefera Belachew ${ }^{2}$ \\ 1 Human Nutrition Unit, School of Public Health, Dilla University, Dilla, Ethiopia \\ 2 Department of Population and family health, College of health Sciences, Jimma University, Jimma, \\ Ethiopia \\ * Correspondence: robelhussen@rocketmail.com
}

\begin{abstract}
Background: Treatment at stabilization center is an important intervention to avert the huge burden of mortality for children with complicated severe acute malnutrition (SAM). Recent reviews indicated a wide range in recovery rate (34-88\%) due to several context-specific factors. This study aimed to estimate time to recovery and to determine predictors of time to recovery among children aged 6-59 month with severe acute malnutrition. Method: Retrospective cohort study was used among 375 children aged 6-59 months admitted in Jimma university medical center, from September 2015 to September 2017. Kaplan Meir estimate and survival curve was used to compare the time to recovery using log-rank test among different characteristics. Cox Proportional Hazard Model was used to identify significant predictors of time to recovery. Results: Median time of recovery for cohort of SAM children's was 19 days (95\%CI: 17.95-20.05). Independent predictors of time to recovery were: Play stimulation, vaccination status, Tuberculosis, malaria, use of amoxicillin, deworming and shock. Conclusion: The findings of this study showed that the average length of stay on treatment and median time for recovery are within the sphere standard. Psychosocial stimulation, appropriate provision of routine medication and management of medical co-morbidity are needed to promote fast recovery.
\end{abstract}

Keywords: Retrospective cohort; predictors; recovery; severe acute malnutrition; Jimma

\section{Introduction}

In acute malnutrition, the amount of one or more macronutrients available to body tissues is inadequate to sustain optimal function. Macronutrient deficiency may result from inadequate diet, poor absorption of ingested nutrients, or the presence of a chronic inflammatory condition that increases requirements for nutrients while promoting a nutrient wasting, catabolic state (1).

Acute childhood malnutrition leads to greater risk of death or disability from common pediatric illnesses such as pneumonia and diarrheal disease. It also shapes long term health by compromising physical and intellectual development (2).

Based on degree of wasting and the presence of edema Acute malnutrition is classified into severe acute malnutrition (SAM) and moderate acute malnutrition (MAM). It is severe acute malnutrition if the wasting is severe (weight for height $<70 \%$ NCHS median or a low MUAC i.e. $<110$ $\mathrm{mm}$ ) or if there is edema. Acute malnutrition is defined as moderate acute malnutrition if the wasting is less severe (weight for height between less than $-2 \mathrm{Z}$ score and $-3 \mathrm{Z}$ score and above); edematous cases were always classified as severe. In many health facilities mortality rate from severe malnutrition was over $20 \%$ at present, which is unacceptable (3). 
Children with SAM are predisposed to serious infections like diarrhea, pneumonia, skin infection, gut bacterial overgrowth and others. Adherence to the protocol in managing children with SAM results in improved recovery. The treatment integrates both nutritional deficiency and medical co-morbidities (4).

Effectiveness of the treatment is evaluated by recovery rate, death rate, average length of stay and weight gain. Thus acceptable level of mortality, recovery rate and default rate should be below $10 \%$, above $75 \%$ and below $15 \%$, respectively (5). However these parameters are still not achieved in many developing countries (6).

Globally acute malnutrition affects nearly 52 million children under the age of five in 2015 . Africa and Asia share more than $90 \%$ of this effect. Severe form of acute malnutrition (SAM) is associated with the death of one million children the age of five each year. Globally, it is estimated that 25-35 million children under the age of five are severely malnourished, with its prevalence ranging from 5 to $30 \%$ In 2015, more than two thirds of all acutely malnourished children under the age of five lived in Asia and more than one quarter lived in Africa. In Africa, 14.1 million children less than five years old were wasted, of which 4.3 million are severely wasted. Sub-Saharan Africa (SSA) accounts for 13.2 million children next to south Asia (27.8 million) of acute malnutrition burden (5). In sub-Saharan Africa alone, there are 137 million children under the age of five, of which 12.3 million were wasted (7).

In Ethiopia, the level of stunting, underweight and wasting was 38.4, 23.6 and 9.9\%, respectively. Among $9.9 \%$ of wasted children, $2.9 \%$ are severely wasted. In Oromia Region the prevalence of stunting, underweight and wasting was 36.5, 22.5 and 10.6 respectively (8). A study done in Jimma Zone on nutritional status of children showed that $14.4 \%$ were underweight, while $33.9 \%$ were stunted and $19.2 \%$ were wasted (9).

In an effort to improve the quality of hospital care for severely malnourished children and reduce case fatality rates, standardized guidelines have been developed by WHO (10) and Ethiopia (3) for SAM patients. Using these guidelines, case fatality rate has been reduced considerably (11). At stabilization center, treatment for complicated SAM children is an important target intervention to avert huge burden of mortality. More than $15 \%$ of SAM children need admission to therapeutic feeding unit. Recent reviews indicated that the recovery rates for inpatient treatment of severe acute malnutrition using the WHO protocol ranged from 33.6\% to 88.4\% (12-14). A study done in Jimma University Medical Center nutritional rehabilitation unit on severe acute malnutrition showed a recovery rate of $77.8 \%(15)$.

Although there are studies on recovery of admitted children with SAM and their predictors (1618), the majority were limited to children below the age of six months, had smaller sample size and unable to clearly show the effects of some factors like season and psychosocial stimulation. As the factors vary according to context, this study aimed to estimate time to recovery and effect of psychosocial stimulation on time to recovery from SAM after adjusting for their contextual factors.

\section{Materials and Methods}

Institution based retrospective cohort study was conducted in Jimma University Medical Center (JUMC).This University is one of the teaching and tertiary medical centers in Ethiopia located in Oromia Region, Jimma Zone; Jimma Town. Jimma Town is located at about 346 kms, Southwest of Addis Ababa. JUMC offers services for nearly 9000 inpatient and 80,000 outpatient attendants in a year within the catchments area covering a $250 \mathrm{~km}$ radius. Severe acute malnutrition is defined as weight for height $<70 \%$ NCHS median or a low MUAC i.e. $<110 \mathrm{~mm}$ or if there is bilateral pitting edema. Recovered (Cured): when the child reaches $>=85 \%$ of median WFH or WFH Z score $>=-2$ on more than one occasion or no edema for 10 days. SAM cases whose card has incomplete data on outcome variable and SAM cases with documented other causes of edema were excluded. This study included all eligible 375 SAM cases. Data were collected from SAM registries and medical records of children. Six graduated diploma nurses were recruited to collect the data. Length of stay and average weight gain were calculated from the available secondary information from cards. We included all cases of children with SAM treated in Jimma University Medical Center nutritional rehabilitation 
unit (NRU) between September 2015 and September 2017 and we excluded those SAM cases whose card has incomplete data on outcome variable, those SAM cases who were readmitted within the study period and those SAM cases with documented other causes of edema. Schematic presentation of sampling procedure is shown in figure 1

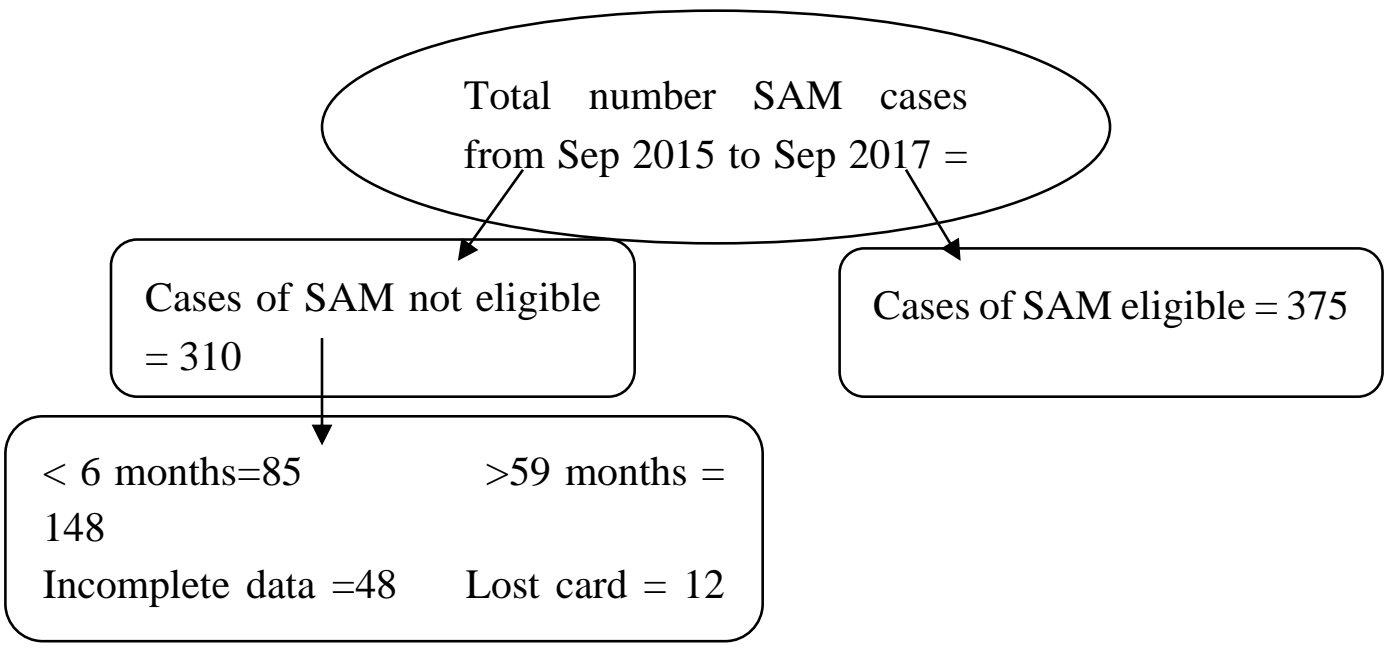

Figure 1. schematic presentation of sampling procedure.

\subsection{Data collection Method}

A structured and pretested data collection tool (format) was used to abstract the data from medical records. Data were abstracted from SAM registries and cards of children retrieved from card room using medical record number. Six graduated diploma nurses were recruited to collect the data from the patient medical record and SAM treatment registry. Before the actual data collection, the data collection tool was pretested and necessary amendments were made. One supervisor with principal investigator followed the data collection closely. Length of stay and average weight gain were calculated from the available secondary information from cards. Anthropometric data of patient and other data were taken from medical cards and SAM registry.

Variables/characteristics at admission: Variable taken as admission variable if recorded or diagnosed within the first 48 hours of admission.

Play stimulation: a play session was held by a trained nurse at decorated play stimulation room with different toys and playing aids based on the age of the child for at least for 30-45min/day. A child will be considered as getting play stimulation if he attended a minimum of 3 play session; otherwise, it is not considered as having play stimuli.

To assure data quality, the data collection tool was pretested on 20 patient cards treated in Jimma university medical center. After pre-test necessary amendment of tool was done for the final data collection. Then the data collection tool was corrected and data collectors were made to aware of changes made. Two days Intensive training was given for six data collector diploma nurses on how to extract the data from patient registry. The data that are collected daily were checked by supervisor and principal investigator for completeness and consistencies.

Data were entered into Epidata version 3.02 to avoid clerical errors.

Variables like anemia were crosschecked by taking recorded measures of hemoglobin from the chart against physician's diagnosis to improve data quality. In the case of play stimulation variable list of children indexed by their medical record number who received play stimulation therapy was taken from play stimulation clinics record in the NRU.

\subsection{Data processing and analysis}


Data were coded and entered to Epidata software version 3.02. Then data were exported to SPSS version 24 for cleaning, checking and analysis. Age, weight, height and edema were further exported to ENA-SMART software to calculate $\mathrm{WFH} \%$ and $\mathrm{HAZ}$ score from admission and discharge measurements. Descriptive statistics using frequency, percent and measure of central tendency was done. Survival curve was also used to compare time to cure by psycho-social stimulation status. The outcome variable was dichotomized as cured and censored for survival analysis. Factors related to time to recovery were analyzed using multivariable Cox proportional hazard model. Proportional hazard assumption of Cox proportional hazard model was checked by plotting log-minus-log survival plot against time for different variables. To control confounding effect of variables, multivariable cox proportional hazard model was used. Variables with $p$ value less than 0.25 in bivariate analysis were selected and included in multivariable analysis. Associations with $P$ value less than 0.05 declared as statistically significant association.

\section{Results}

\subsection{Socio demographic and care related characteristics}

A cohort of 375 SAM children were followed retrospectively for median time of 17 days with Inter quartile range of 10 days. From this, 191 (50.9\%) were females while the rest are males. The mean age (in months) of study subjects was 26.8 month with standard deviation of 14.6 month with more than half $(53 \%)$ are between one and three years. Two hundred fifty five $(68 \%)$ were from rural areas. Nearly one third $107(28.5 \%)$ of the children were admitted on winter (June to august) season. Nearly two-third of children $238(63.5 \%$ ) were fully vaccinated for their age (table 1).

Table 1. Socio Demographic and care related characteristics of SAM children admitted in JUMC, nutritional rehabilitation unit from sept.2015 to sept.2017.

\begin{tabular}{|c|c|c|}
\hline Characteristics & Number & Percent $(\%)$ \\
\hline \multicolumn{3}{|l|}{ Age (in months) $n=375$} \\
\hline 6-11 & 55 & 14.7 \\
\hline $12-23$ & 110 & 29.3 \\
\hline 24-35 & 89 & 23.7 \\
\hline $36-47$ & 61 & 16.3 \\
\hline 48-59 & 60 & 16 \\
\hline \multicolumn{3}{|c|}{ Sex of the respondent $(n=375)$} \\
\hline Female & 191 & 50.1 \\
\hline Male & 184 & 49.9 \\
\hline \multicolumn{3}{|l|}{ Residence ( $\mathrm{n}=375$ ) } \\
\hline Urban & 120 & 32 \\
\hline Rural & 255 & 68 \\
\hline \multicolumn{3}{|l|}{ Season of admission $(\mathrm{n}=375)$} \\
\hline Winter & 107 & 28.5 \\
\hline Summer & 85 & 22.7 \\
\hline Spring & 89 & 23.7 \\
\hline Autumn & 94 & 25.1 \\
\hline \multicolumn{3}{|c|}{ Vaccination status for age $(\mathrm{n}=375)$} \\
\hline Fully vaccinated & 238 & 63.5 \\
\hline Partially vaccinated & 50 & 13.3 \\
\hline
\end{tabular}




\begin{tabular}{lcc}
\hline Not vaccinated & 36 & 9.6 \\
Not documented & 51 & 13.6 \\
Exclusive breast feeding $(\mathrm{n}=322)$ & & \\
Yes & 196 & 52.5 \\
No & 126 & 33.6 \\
\hline
\end{tabular}

\subsection{Anthropometry and complication related characteristics}

More than half $(65.9 \%)$ of the children have edematous SAM and $224(59.7 \%)$ passed appetite test. Over two third $(66.6 \%)$ of the children were stunted while nearly half $(48.3 \%)$ had WFH \% median below $70 \%$ (Table 2 ).

Table 2. Baseline anthropometry related characteristics of SAM children admitted in JUMC, nutritional rehabilitation unit from sept.2015 to sept.2017.

\begin{tabular}{|c|c|c|}
\hline Variable & Number & Percent \\
\hline \multicolumn{3}{|l|}{ Nutritional edema } \\
\hline Yes & 247 & 65.9 \\
\hline No & 128 & 34.1 \\
\hline \multicolumn{3}{|l|}{ Appetite test } \\
\hline Failed & 151 & 40.3 \\
\hline Passed & 224 & 59.7 \\
\hline \multicolumn{3}{|l|}{ WFH \% } \\
\hline$<70$ & 181 & 48.3 \\
\hline $70-80$ & 23 & 6.1 \\
\hline$>80$ & 171 & 45.6 \\
\hline \multicolumn{3}{|l|}{ Chronic nutritional status } \\
\hline Stunted $($ HAZ<-2 SD) & 219 & 66.6 \\
\hline Not Stunted (HAZ >-2SD) & 110 & 33.4 \\
\hline
\end{tabular}

Three hundred fifty six (94.9\%) had at least one type of complication at admission. As shown in (Table 3) diarrhea and anemia were the most common co morbidities among admitted SAM children. About 268 (71.4\%) and 159 (42.4\%) had diarrhea and anemia respectively as a major co morbidity. These cases were followed by pneumonia (29.6\%), dehydration (14.7\%), tuberculosis (14.4\%), malaria (5.6\%) and HIV (3.5\%), respectively. Twenty five (6.7\%) of them had septic or hypo-volumic shock at admission. And about 304(81.1\%) were conscious at admission.

Table 3. Medical complication and clinical features of SAM children admitted in JUMC, nutritional rehabilitation unit from sept.2015 to sept.2017.

\begin{tabular}{|llll|}
\hline \multicolumn{1}{c}{ Variable } & Category & Number & Percent \\
\hline Complication at admission & Yes & 356 & 94.9 \\
\hline
\end{tabular}




\begin{tabular}{|c|c|c|c|}
\hline & No & 19 & 5.1 \\
\hline \multirow[t]{2}{*}{ Diarrhea } & Yes & 268 & 71.5 \\
\hline & No & 88 & 23.5 \\
\hline \multirow[t]{2}{*}{ Tuberculosis } & Yes & 54 & 14.4 \\
\hline & No & 302 & 85.6 \\
\hline \multirow[t]{2}{*}{ HIV $(n=190)$} & Yes & 15 & 3.5 \\
\hline & No & 175 & 91.9 \\
\hline \multirow[t]{2}{*}{ Pneumonia } & Yes & 111 & 29.6 \\
\hline & No & 245 & 65.3 \\
\hline \multirow[t]{2}{*}{ Anemia } & Yes & 159 & 42.4 \\
\hline & No & 197 & 52.5 \\
\hline \multirow[t]{2}{*}{ Malaria } & Yes & 21 & 5.6 \\
\hline & No & 335 & 89.3 \\
\hline \multirow[t]{2}{*}{ Dehydration } & Yes & 55 & 14.7 \\
\hline & No & 301 & 80.3 \\
\hline \multirow[t]{2}{*}{ Shock } & Yes & 25 & 6.7 \\
\hline & No & 350 & 93.3 \\
\hline \multirow[t]{2}{*}{ Consciousness } & Yes & 304 & 81.1 \\
\hline & No & 71 & 18.9 \\
\hline
\end{tabular}

\subsection{Medication and nutritional therapy related characteristics}

With regard to routine medication and nutritional therapy $299(79.4 \%)$ of them had taken folic acid and 270(72\%) of them took amoxicillin. While 158(42.1\%), 126(33.6\%), 129(34.4\%) and 161(42.9) took vitamin $\mathrm{A}$, iron, deworming and special medication, respectively. Two hundred ninety six $(78.9 \%)$ had taken IV antibiotic and $68.1(18.1 \%)$ has taken IV fluid therapy. A few 30(8\%) had blood transfusion and 130(34.4\%) used NG tube. Almost all 364(97.1\%) has got formula 75 nutritional therapy while 293(78.1\%), 237(63.2\%) received formula 100 and plumpy nut therapy, respectively. Seventy four $(19.7 \%)$ of them children also received play stimulation.

\subsection{Survival pattern and time to recovery}

Regarding the outcome of the treatment of cohort of children with SAM, 274(73.1\%, 95\% CI: 68.577.4) recovered while $46(12.35,95 \%$ CI: 9.06-15.6), $10(2.7 \%, 95 \%$ CI: $1.05-4.35)$ and $45(12 \%, 95 \%$ CI: 8.71-15.28) died, transferred out and defaulted, respectively (figure 2). 


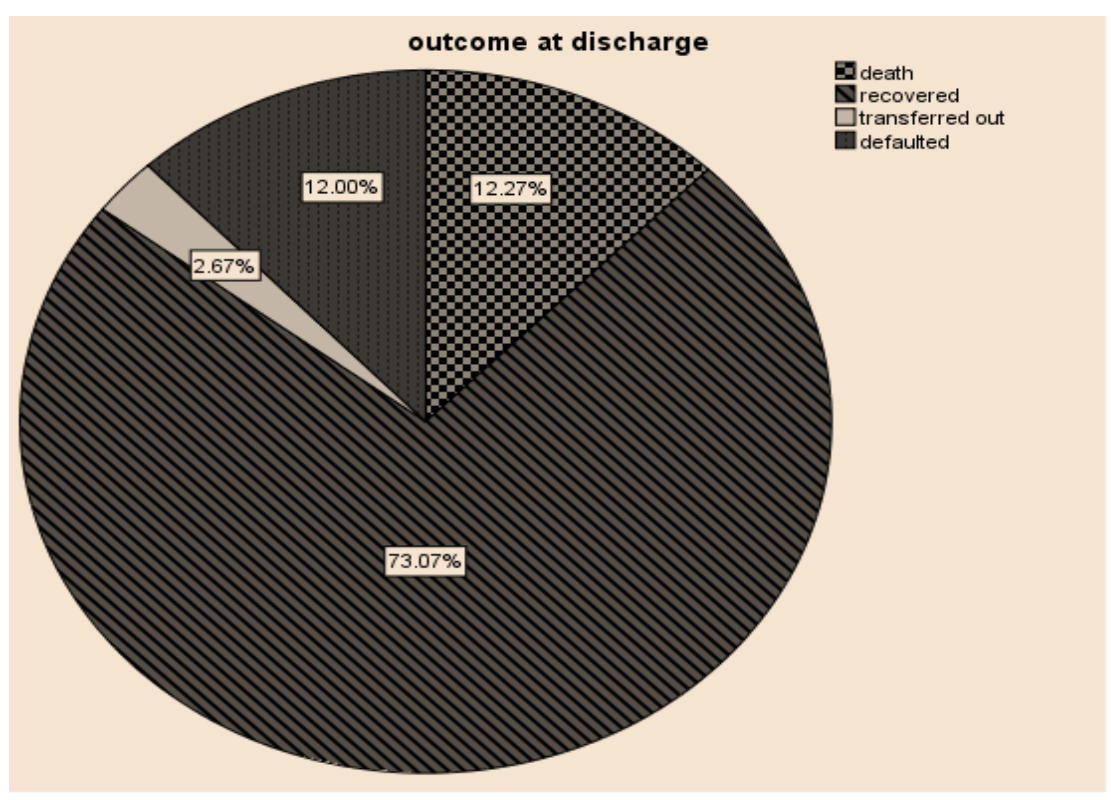

Figure 2. Treatment outcome of admitted SAM children in Jimma University Medical Center between September 2015 and September 2017, Jimma.

The overall follow up time for 375 SAM children was 6748 days with cumulative incidence of recovery 0.0406 recovery per person day (40.6 recovery /1000 person days) among admitted children.

Table 4. Actuarial Life table analysis showing survival of SAM children admitted in JUMC from 2015 to 2017, Jimma, Southwest Ethiopia.

\begin{tabular}{|c|c|c|c|c|c|c|c|}
\hline $\begin{array}{c}\text { Interval } \\
\text { Start } \\
\text { Time }\end{array}$ & $\begin{array}{l}\text { Number } \\
\text { Entering } \\
\text { Interval }\end{array}$ & $\begin{array}{l}\text { Number } \\
\text { censored }\end{array}$ & $\begin{array}{c}\text { Number } \\
\text { Exposed } \\
\text { to Risk }\end{array}$ & $\begin{array}{l}\text { Number } \\
\text { recovered }\end{array}$ & $\begin{array}{c}\text { Proportion } \\
\text { not } \\
\text { recovered }\end{array}$ & $\begin{array}{l}\text { Proport } \\
\text { ion } \\
\text { Survivi } \\
\text { ng }\end{array}$ & $\begin{array}{c}\text { Cumulative } \\
\text { Proportion } \\
\text { recovered }\end{array}$ \\
\hline $\mathbf{0}$ & 375 & 12 & 369 & 1 & .00 & 1.00 & 1.00 \\
\hline 5 & 362 & 36 & 344 & 3 & .01 & .99 & .99 \\
\hline 10 & 323 & 23 & 311.5 & 66 & .21 & .79 & .78 \\
\hline 15 & 234 & 17 & 225.5 & 89 & .39 & .61 & .47 \\
\hline 20 & 128 & 5 & 125.5 & 47 & .37 & .63 & .30 \\
\hline 25 & 76 & 2 & 75 & 37 & .49 & .51 & .15 \\
\hline 30 & 37 & 1 & 36.5 & 18 & .49 & .51 & .08 \\
\hline 35 & 18 & 1 & 17.5 & 7 & .40 & .60 & .05 \\
\hline 40 & 10 & 2 & 9 & 4 & .44 & .56 & .03 \\
\hline 45 & 4 & 1 & 3.5 & 1 & .29 & .71 & .02 \\
\hline 50 & 2 & $\mathbf{0}$ & 2 & 1 & .50 & .50 & .01 \\
\hline 55 & 1 & 1 & .5 & $\mathbf{0}$ & .00 & 1.00 & .01 \\
\hline
\end{tabular}




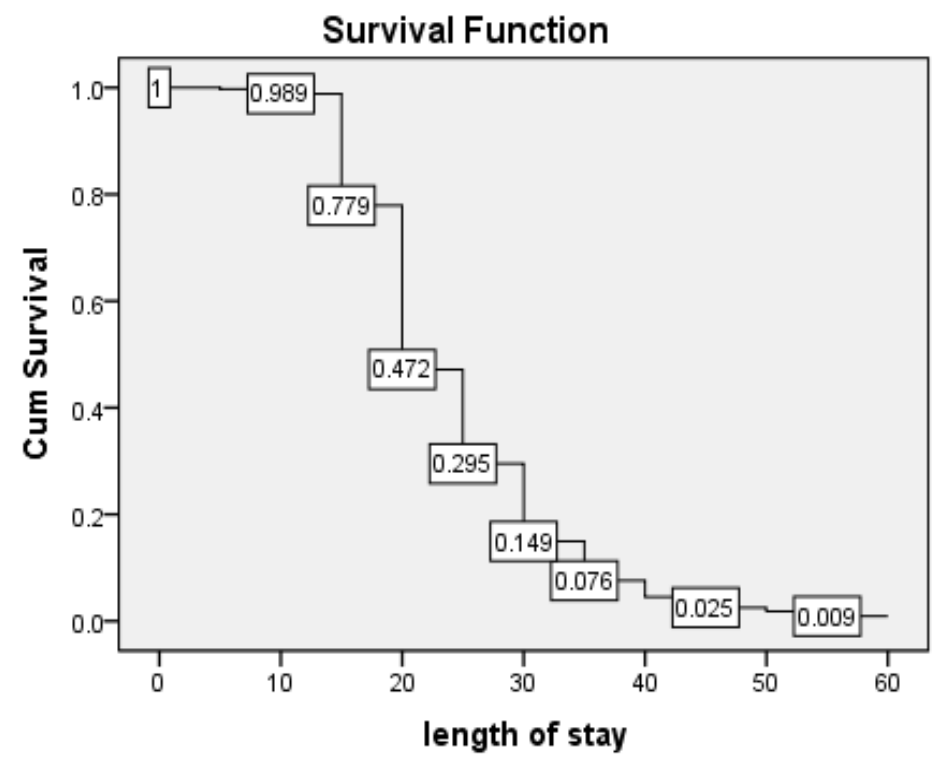

Figure 3. Depicted survival graph for length of stay (days) of entire cohort of children admitted with SAM in JUMC from 2015 to 2017, Jimma, Ethiopia.

Table 4 and figure 3 illustrate median time of recovery from SAM among children with SAM managed at NRU. The figure revealed that the overall median length of stay for the entire cohorts of children with SAM was 17 days \pm 10 days of inter quartile range. The mean length of stay for the entire cohort is 18 days with minimum of one day and maximum of 56 days. Actuarial Life Table analysis showed that cumulative probability of nutritional recovery was $99,78,30,8$, and $1 \%$ at $5,10,15,20$, 30 and 50 days, respectively.

Figure 4 illustrates Kaplan Meir survival curve showing the effect of for psychosocial stimulation of children with severe acute malnutrition on time to discharge

\subsection{Predictors of time to recovery}

Bivariate analysis was performed for the variables such as, socio-demographic characteristics, anthropometry, type of malnutrition, complication at admission, clinical features, type of medications and nutritional therapy using bivariate Cox regression. Statistically significance was observed in children with play stimulation, categories of age, season of admission, exclusive breast feeding, vaccination status, type of SAM, edema, complication at admission, TB, HIV, malaria, shock, appetite, complication after admission, folic acid, amoxicillin, ampicillin, deworming, gentamicin, special medication, blood transfusion, IV antibiotic, IV fluid, NG tube use and plumpy nut, F-100 $\mathrm{a}(\mathrm{P}<0.25)$.(Table 5)

Table 5. Bivariate Cox proportional hazard Model for predictors of time to recovery from SAM among Children admitted in Jimma university medical center, Jimma, southwest Ethiopia.

\begin{tabular}{|c|c|c|c|c|c|}
\hline & Variable & Cured & Censored & $\begin{array}{l}\text { Crude Hazard } \\
\text { ratio } \\
\text { With } 95 \% \mathrm{CI}\end{array}$ & $\mathbf{P}$ \\
\hline \multicolumn{6}{|c|}{ Socio demographic characteristics } \\
\hline Sex & Male & 140 & 51 & $0.98(0.77-1.28)$ & 0.888 \\
\hline
\end{tabular}




\begin{tabular}{|c|c|c|c|c|c|c|}
\hline & Female & 134 & 50 & 1 & & \\
\hline \multirow[t]{5}{*}{ Age(months) } & $6-11$ & 37 & 18 & $0.69(0.45-1.05)$ & \multicolumn{2}{|l|}{$0.086^{*}$} \\
\hline & $12-23$ & 78 & 32 & $0.59(0.42-0.85)$ & \multicolumn{2}{|l|}{$0.004 *$} \\
\hline & $24-35$ & 68 & 21 & $0.78(0.54-1.13)$ & \multicolumn{2}{|l|}{$0.19^{*}$} \\
\hline & $36-47$ & 41 & 20 & $0.42(0.28-0.65)$ & \multicolumn{2}{|l|}{$0.001^{*}$} \\
\hline & $48-59$ & 50 & 10 & 1 & & \\
\hline \multirow[t]{2}{*}{ Residence } & Urban & 89 & 31 & $0.94(0.73-1.22)$ & \multirow{2}{*}{\multicolumn{2}{|c|}{0.659}} \\
\hline & Rural & 185 & 70 & 1 & & \\
\hline \multirow[t]{4}{*}{ Season } & Winter & 77 & 30 & $1.39(1.01-1.9)$ & \multicolumn{2}{|l|}{$0.042 *$} \\
\hline & Summer & 52 & 33 & $1.39(0.97-1.98)$ & \multicolumn{2}{|l|}{$0.07 *$} \\
\hline & Spring & 67 & 22 & $1.29(0.89-1.72)$ & \multicolumn{2}{|l|}{$0.205^{*}$} \\
\hline & Autumn & 78 & 16 & 1 & & \\
\hline \multirow[t]{2}{*}{ Exclusive breast feeding } & Yes & 147 & 50 & $0.81(0.62-1.07)$ & \multirow{2}{*}{\multicolumn{2}{|c|}{$0.133^{*}$}} \\
\hline & No & 84 & 42 & 1 & & \\
\hline \multirow[t]{4}{*}{ Vaccination } & Fully vaccinated & 183 & 55 & $1.39(0.9-2.14)$ & \multicolumn{2}{|l|}{$0.13 *$} \\
\hline & Partially vaccinated & 28 & 22 & $1.12(0.65-1.93)$ & \multicolumn{2}{|l|}{0.68} \\
\hline & Unknown & 39 & 12 & $1.16(0.69-1.93)$ & \multicolumn{2}{|l|}{0.57} \\
\hline & Not vaccinated & 24 & 12 & 1 & & \\
\hline \multicolumn{7}{|c|}{ Type of malnutrition, complication and clinical features } \\
\hline \multirow[t]{4}{*}{ Type of SAM } & Marasmus & 94 & 34 & $1.59(1.095-2.32)$ & \multicolumn{2}{|l|}{$0.015^{*}$} \\
\hline & Kwashiorkor & 141 & 51 & $1.51(1.06-2.16)$ & \multicolumn{2}{|l|}{$0.022 *$} \\
\hline & Marasmic- & 39 & 16 & 1 & & \\
\hline & kwashiorkor & & & & & \\
\hline \multirow[t]{2}{*}{ Edema } & Yes & 180 & 67 & $0.856(0.67-1.02)$ & \multirow{2}{*}{\multicolumn{2}{|c|}{$0.224 *$}} \\
\hline & No & 94 & 34 & 1 & & \\
\hline \multirow{2}{*}{$\begin{array}{l}\text { Complication } \\
\text { admission }\end{array}$} & Yes & 257 & 99 & $0.64(0.39-1.04)$ & \multirow{2}{*}{\multicolumn{2}{|c|}{$0.071 *$}} \\
\hline & No & 17 & 2 & 1 & & \\
\hline \multirow[t]{2}{*}{ Diarrhea } & Yes & 191 & 77 & 1 & \multirow{2}{*}{\multicolumn{2}{|c|}{0.433}} \\
\hline & No & 66 & 22 & $0.89(0.67-1.18)$ & & \\
\hline TB & Yes & 29 & 25 & 1 & & \\
\hline
\end{tabular}




\begin{tabular}{|c|c|c|c|c|c|c|}
\hline & No & 228 & 74 & $1.72(1.17-2.5)$ & $0.006^{*}$ & \\
\hline \multirow[t]{2}{*}{ HIV } & Yes & 5 & 10 & 1 & \multirow{2}{*}{$0.018^{*}$} & \\
\hline & No & 254 & 89 & $3.96(1.26-12.4)$ & & \\
\hline \multirow[t]{2}{*}{ Pneumonia } & Yes & 76 & 35 & 1 & \multirow[b]{2}{*}{0.411} & \\
\hline & No & 181 & 64 & $0.89(0.68-1.17)$ & & \\
\hline \multirow[t]{2}{*}{ Anemia } & Yes & 115 & 44 & 1 & & \\
\hline & No & 142 & 55 & $1.1(0.86-1.4)$ & 0.467 & \\
\hline \multirow[t]{2}{*}{ Malaria } & Yes & 14 & 7 & 1 & & \\
\hline & No & 243 & 92 & $1.86(1.08-3.2)$ & $0.024 *$ & \\
\hline \multirow[t]{2}{*}{ Dehydration } & Yes & 32 & 23 & 1 & \multirow{2}{*}{0.428} & \\
\hline & No & 225 & 76 & $1.16(0.8-1.68)$ & & \\
\hline \multirow[t]{2}{*}{ Shock } & Yes & 4 & 21 & 1 & \multirow{2}{*}{$0.029 *$} & \\
\hline & No & 270 & 80 & $3.02(1.12-8.12)$ & & \\
\hline \multirow[t]{2}{*}{ Consciousness } & Impaired & 33 & 38 & 1 & \multirow[b]{2}{*}{0.272} & \\
\hline & Conscious & 241 & 63 & $1.23(0.85-1.77)$ & & \\
\hline \multirow[t]{2}{*}{ Appetite test } & Failed & 96 & 55 & 1 & \multirow{2}{*}{$0.008^{*}$} & \\
\hline & Passed & 178 & 46 & $1.4(1.09-1.81)$ & & \\
\hline \multirow{2}{*}{$\begin{array}{l}\text { Co-morbidity } \\
\text { admission }\end{array}$} & Yes & 41 & 25 & 1 & \multirow{2}{*}{$0.052 *$} & \\
\hline & No & 233 & 76 & $1.39(0.99-1.94)$ & & \\
\hline \multicolumn{7}{|c|}{ Medication and nutritional therapy related characteristics } \\
\hline \multirow[t]{2}{*}{ Vitamin A } & Yes & 118 & 40 & $1.05(0.83-1.34)$ & \multirow[t]{2}{*}{0.688} & \\
\hline & No & 156 & 61 & 1 & & \\
\hline \multirow[t]{2}{*}{ Folic acid } & Yes & 221 & 78 & $1.45(1.07-1.97)$ & & $0.015^{*}$ \\
\hline & No & 53 & 23 & 1 & & \\
\hline \multirow[t]{2}{*}{ Amoxicillin } & Yes & 207 & 63 & $1.32(1-1.74)$ & \multirow{2}{*}{$0.049 *$} & \\
\hline & No & 67 & 38 & 1 & & \\
\hline \multirow[t]{2}{*}{ Ampicillin } & Yes & 173 & 81 & $0.84(0.66-1.07)$ & & $0.164 *$ \\
\hline & No & 101 & 20 & 1 & & \\
\hline Iron & Yes & 109 & 17 & $1.14(0.89-1.45)$ & & \\
\hline
\end{tabular}




\begin{tabular}{|c|c|c|c|c|c|c|}
\hline & No & 165 & 84 & 1 & 0.298 & \\
\hline \multirow[t]{2}{*}{ Deworming } & Yes & 110 & 19 & $1.22(0.96-1.55)$ & \multirow{2}{*}{$0.107 *$} & \\
\hline & No & 164 & 82 & 1 & & \\
\hline \multirow[t]{2}{*}{ Gentamicin } & Yes & 201 & 80 & $0.7(0.54-0.92)$ & \multirow{2}{*}{$0.012 *$} & \\
\hline & No & 73 & 21 & 1 & & \\
\hline \multirow[t]{2}{*}{ Special medication } & Yes & 104 & 57 & $0.58(0.45-0.74)$ & \multirow{2}{*}{\multicolumn{2}{|c|}{$0.0000 *$}} \\
\hline & No & 170 & 44 & 1 & & \\
\hline \multirow[t]{2}{*}{ Blood transfusion } & Yes & 6 & 24 & 1 & \multirow[b]{2}{*}{$0.001 *$} & \\
\hline & No & 268 & 77 & $4(1.77-9.07)$ & & \\
\hline \multirow[t]{2}{*}{ IV antibiotic } & Yes & 209 & 87 & $0.67(0.5-0.89)$ & \multirow{2}{*}{\multicolumn{2}{|c|}{$0.005 *$}} \\
\hline & No & 65 & 14 & 1 & & \\
\hline \multirow[t]{2}{*}{ IV fluid } & Yes & 24 & 44 & $0.45(0.29-0.69)$ & \multirow{2}{*}{\multicolumn{2}{|c|}{$0.000 *$}} \\
\hline & No & 250 & 57 & 1 & & \\
\hline \multirow[t]{2}{*}{ Plumpy nut intake } & Yes & 208 & 29 & $1.74(1.3-2.3)$ & \multirow{2}{*}{\multicolumn{2}{|c|}{$0.000^{*}$}} \\
\hline & No & 66 & 72 & 1 & & \\
\hline \multirow[t]{2}{*}{ NG tube } & Yes & 74 & 56 & $0.67(0.5-0.88)$ & \multirow{2}{*}{\multicolumn{2}{|c|}{$0.004 *$}} \\
\hline & No & 200 & 45 & 1 & & \\
\hline \multirow[t]{2}{*}{ F-100 } & Yes & 251 & 42 & $1.75(1.14-2.68)$ & \multirow{2}{*}{\multicolumn{2}{|c|}{$0.011^{*}$}} \\
\hline & No & 23 & 59 & 1 & & \\
\hline \multirow[t]{2}{*}{ F-75 } & Yes & 268 & 96 & $0.84(0.37-1.9)$ & \multirow{2}{*}{\multicolumn{2}{|c|}{0.678}} \\
\hline & No & 6 & 5 & 1 & & \\
\hline \multirow[t]{2}{*}{ Play stimulation } & Yes & 72 & 2 & 1.27 & \multirow{2}{*}{\multicolumn{2}{|c|}{$0.083^{*}$}} \\
\hline & No & 202 & 99 & 1 & & \\
\hline
\end{tabular}

\subsubsection{Multivariable analysis:}

Multivariable Cox regression analysis was performed for variables identified in the bivariate Cox regression as significant $(\mathrm{p}<0.25)$ by adjusting for confounders through step wise backward multivariable Cox regression method.

Vaccination status (fully vaccinated), play stimulation, $\mathrm{TB}$, malaria, amoxicillin, deworming and shock were found to be independent predictors of nutritional recovery time. The likelihood of early recovery in fully vaccinated children was $2.26(\mathrm{AHR}=2.26,95 \% \mathrm{CI}: 1.12-4.57)$ times those who were not vaccinated. Similarly, children who received play stimulation therapy were $1.93(\mathrm{AHR}=1.93$, 95\%CI: 1.23-3.03) times likely to recover fast. Likewise, SAM children with TB were 52\% (AHR=0.48, 95\% CI: 0.27-0.87) who are more likely to have a delayed recovery time compared to children without 
TB. Besides, children with malaria $65.9 \%$ (AHR=0.34, 95\%CI: $0.13-0.88$ ) have more probability of delayed recovery than their counterparts. In addition, children who took amoxicillin were at $1.54 \%$ ( $\mathrm{AHR}=1.54$, 95\%CI: 0.008-2.34) times more likelihood of fast recovery than their counterparts. Children with SAM who took deworming were $1.8 \%(\mathrm{AHR}=1.8,95 \% \mathrm{CI}$ : 1.18-2.73) times more likely to have early recovery than children without deworming. The likelihood of delayed recovery is $82 \%$ $(\mathrm{AHR}=0.18$, 95\%CI: 0.05-0.59) more in those who developed shock relative to those who did not develop shock (Table 6).

Table 6. Multivariate Cox proportional Hazard Regression model for predictors of time to recovery from SAM among Children admitted in Jimma university medical center, Jimma, southwest Ethiopia.

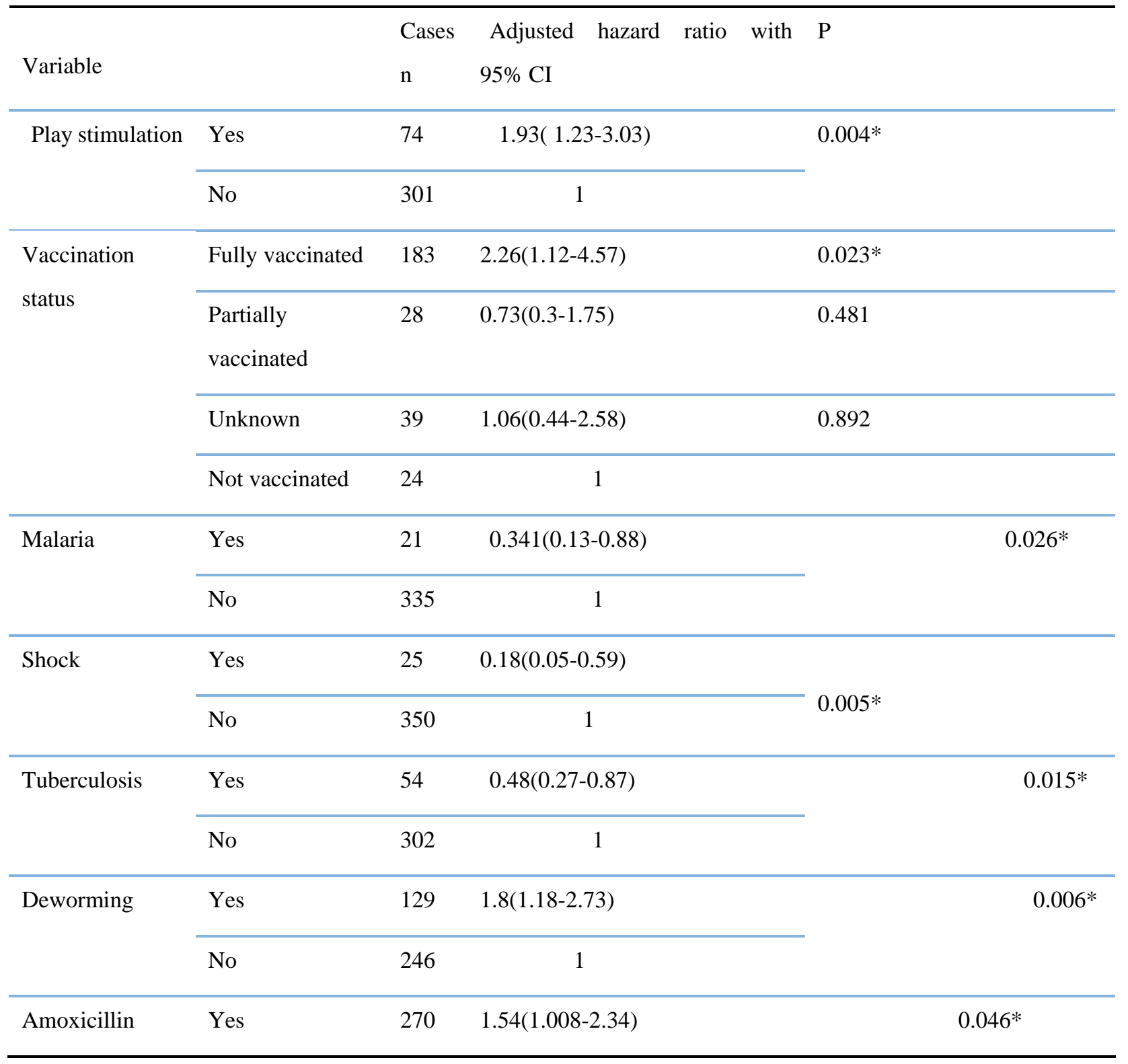

\section{Discussion}

This study revealed important information about nutritional recovery time and its predictors on children with SAM managed in nutritional rehabilitation unit. The overall median length of stay of the entire cohort was 17 days \pm 9 days and median nutritional recovery time for cohort of SAM children was observed to be 19 days both are within the accepted national minimum standards of average length of stay for inpatient treatment (3). This result was in line with other studies conducted in northern Ethiopia and other developing countries (19). However, in contrast to studies conducted 
in southern Ethiopia $(11,16)$, this study found a shorter recovery time. This may be due to difference in implementation of SAM management guideline (3), staffing and setting. Looking at the type of SAM, edematous malnutrition accounted for more than half 247(65.9\%). This was in line with studies done in Ethiopia and other African countries (11, 15, and 20). The high prevalence of edematous SAM may be due to frequent intake of carbohydrate and low intake of protein rich foods (20). Diarrhea is the most common co-morbidity seen in $268(71.5 \%)$ children which is consistent with reports from studies 70.3 in Kenya (21), 44.6\% in Sekota (13) and 63.4\% in Mekelle (17).

Regarding treatment outcome, the minimum acceptable standard of sphere project is $>75 \%$ recovery, $<15 \%$ default rate, $<10 \%$ death rate (5). In this study the recovery rate is $73.1 \%$ which is in line with the minimum standard. This result is greater than the reports from Gondar University Referral Hospital (22), Mekelle (17) and Bahirdar (23). In contrast to study done in Southern Ethiopia therapeutic feeding centers they reported that the nutritional recovery rate was 3.61 per 100 person day observations. Rate of recovery in this study was 4.06 per 100 person days (40.6 recovery /1000 person days). This difference can be attributed to the existence of high level professionals like pediatricians and pediatric residents in this study setup.

Our findings indicated that children who were provided with a combination of psychosocial stimulation and therapeutic feeding tended to gain weight at a faster rate of 1.93 times more likely to recover than those who received only therapeutic feeding. Even if play stimulation was included in Ethiopian SAM management guideline, it was not common to see the service in health institutions except in Jimma University Medical Center. The introduction of play stimulation therapy to the existing SAM management has a rewarding effect via reducing hospital stay. This shortened hospital stay has a direct implication in terms of cost and quality of SAM management. Although further studies are required to substantiate, the findings call for arguments to include this very important, but neglected component of treating children with SAM. In addition to dietary and medical therapy play stimulation therapy has to be given in all other health facilities in the country.

Among socio-demographic characteristics, vaccination status is independent predictor of nutritional recovery time. In this study, children who are not fully vaccinated for age took 2.26 longer recovery time compared to their fully vaccinated for age counter parts. Similarly, a study conducted in Felegehiwot Referral Hospital reported that fully vaccinated SAM children had 4.12 times better recovery rate than their unvaccinated counter parts (23). This can be explained by the role of vaccination in preventing several contagious diseases.

Findings from play therapy in Africa (24) indicated that play stimulation increased speed of recovery. Children who received play therapy were discharged as cured before the end of the $4^{\text {th }}$ and the $5^{\text {th }}$ week from admission compared to the control groups who are not received play stimulation who were not discharged before the end of $6^{\text {th }}$ week. Besides another study conducted in Bangladesh reported that severely malnourished children who received play stimulation have improved weight for age Z-score than their counterparts (25). Results from a randomized placebo controlled trial conducted in Southern Malawi on SAM children showed that nutritional recovery was 1.38 times greater for those who took amoxicillin than the placebo group and it also indicated nutritional recovery time was shorter for those who took amoxicillin than the placebo group (26). Similarly, this study found the fact that amoxicillin speed up recovery. Children who took amoxicillin found to have $54 \%$ (AHR $=1.54,95 \%$ CI: 1.008-2.34) increased speed of recovery than those children who did not take. Recent updates of WHO (10) and national guideline for SAM management (3) recommend provision of broad spectrum antibiotic like amoxicillin for all severely malnourished children regardless of signs of infection and complications. Deworming was also found to be predictor of short recovery time. Severely malnourished children who took albendazole/mebendazole were 1.8 times more likely to have shorter recovery time as compared to those children who did not (AHR=1.8, 95\% CI: 1.18-2.73). This may be due to high prevalence of intestinal parasite in severely malnourished children which results in reduced appetite and nausea in children who do not get deworming.

The hazard of death due to TB was nearly three times higher than children with no

$\mathrm{TB}(\mathrm{AHR}=2.88,95 \% \mathrm{CI}=1.72,4.65)$ as reported by Kebede (13). In this study; however, it was found that among children who had tuberculosis nutritional recovery time was delayed by $51.7 \%$. This can 
be explained by diminished immunity of SAM children which predisposes them to tuberculosis resulting in disease and inflammatory response. This in turn worsens the nutritional state thus delaying the recovery time. This may highlight the vicious cycle of malnutrition and disease; however the mechanism underlying the association between tuberculosis and malnutrition remains unclear (27).

Malaria is among the common febrile illness which affects children. Its effect is highly devastating in severely malnourished children. Even if there is a shortage of studies showing the impact of malaria on recovery rate and recovery time a study done in Sekota Hospital (13) showed that malaria increases death rate and shortens time to death ( $\mathrm{AHR}=2.13,95 \% \mathrm{CI}: 1.12-7.15)$. However, this study revealed that those severely malnourished children with malaria have $65.9 \%$ delayed recovery than their non malarious counter parts. This could be due to the febrile nature of the disease which puts the child in catabolic state this in turn may worsen the child's nutritional state and also may be via reduced appetite.The above mentioned possible reasons thus could lengthen the time needed to recover.

A study done in therapeutic feeding centers in Gedeo Zone showed that development of shock significantly increased the hazard of death by 3.8 times more (28). But the finding of this study shows that children who developed shock had $82 \%$ longer nutritional recovery time than those who did not develop. The possible explanation may be in children who developed shock there is a decreased perfusion to vital organs that may lead to end organ damage, thus prolonging the nutritional recovery time.

A study done in Woldia Hospital indicated that severely malnourished children having HIV/AIDS co-morbidity were $90 \%$ less likely to be cured as compared to those without HIV/AIDS comorbidity. HIV infection was a predominant factor that compromised recovery rate and increased mortality rate. (29) There are also other studies that support the finding that being co morbid with HIV reduces the likelihood of recovery (6 and 10).However, in this study HIV is not a significant predictor of nutritional recovery time $(p>0.05)$. This may be due to shift in practice from routine provider initiated HIV counseling and testing (PIHCT).

SAM children who did not get vaccination for their age have a longer hospital stay so; implementation of the extended immunization program should be strengthened. The results pose arguments for integration of play stimulation therapy in the management of severe acute malnutrition at scale level to prevent childhood mortality thereby to achieve sustainable development goal. Provision and use of routine medications like amoxicillin and deworming, management of complication like shock, TB and malaria should also stick to national guideline for management of SAM. All the aforementioned results showed reduced hospital stay thus, implying reduction in the cost needed for treatment and burden of health institutions; which will in turn ensures quality of care.

Longitudinal nature of the study design, giving an insight for researchers who wish to use a prospective design and addressing the effect of variables like season of admission, vaccination status and play stimulation were some of the strength of this study. However, difficulty ascertaining the reliability of recorded data, potential bias due to excluded records and unknown status of defaulters, and failing to address variables like educational status, house hold wealth index, socioeconomic status, maternal nutritional status, and child's feeding practice that might have affect on recovery, were some of the limitation of the study.

\section{Conclusions}

Based on the findings of this study, average length of stay for the entire cohort and median time of recovery was within the sphere standard. The probability of recovery decreases with increased hospital stay. Play stimulation, vaccination, amoxicillin and deworming were independent predictors of short nutritional recovery while, the presence of malaria, TB and shock were found to be independent predictors of delayed nutritional recovery time. Appropriate provision of routine medication and management of medical co morbidity as per the national SAM management protocol enhances fast recovery. 
Author Contributions: RH: GZ and TB originated the research question, participated in data analysis and data curation, interpretation of the findings and writing of the manuscript. All authors have read and approved the final version of the manuscript.

Funding: This research received no external funding.

Acknowledgments: We would like to acknowledge Jimma University Medical Center who allowed us to undertake this study. Lastly we are extremely grateful to participant involved in the study, data collectors and the rest research team members.

Conflicts of Interest: The authors declare no conflict of interest.

ACRONYMS/ABBREVIATIONS

AIDS Acquired Immune Deficiency Syndrome AHR Adjusted Hazard Ratio

CI Confidence Interval F 75 Formula Milk Seventy Five

F 100 Formula Milk Hundred HIV Human Immune Deficiency Virus

JUMC Jimma University Medical Center MRN Medical Registration Number

MUAC Mid Upper Arm Circumference NRU Nutritional Rehabilitation Unit

OR Odds Ratio RUTF Ready to Use Therapeutic Food

SAM Severe Acute Malnutrition SPSS Statistical Package for Social Sciences

TFC Therapeutic Feeding Center TFU Therapeutic Feeding Unit

TB Tuberculosis UNAIDS United Nations on HIV/AIDS

UNICEF United Nations International Children's Emergency Fund

WFH/A Weight for Height/Age WHO World Health Organization

\section{References}

1. Pelletier DL, Frongillo EA. Changes in Child Survival Are Strongly Associated with Changes in Malnutrition in Developing Countries. Journal of Nutrition, 2003; 133(1):107-19.

2. Victora CG, L A, C F, HallalP C, Martorell R, Richter P, et al. Maternal and child under nutrition: consequences for adult health and human capital. Lancet, 2008; 371:340-57.

3. Federal ministry of health. Protocol for the management of severe acute malnutrition. Addis Ababa,Ethiopia,2007:1-120. Thttps:/files.ennonline.net/attachments/897/ethiopia-sam-guideline-march2007.pdf

4. Hobbs B, Bush A. A 10 point plan for tackling acute malnutrition in under fives. Acute malnutrition an everyday's emergency 2015. https:/www.popline.org/node/579688

5. The sphere project Humanitarian Charter and Minimum Standards in Humanitarian Response. Minimum Standards in Food Security and nutrition: Management of acute malnutrition and micronutrient deficiencies, 
2011: 168-69. http://www.spherehandbook.org/en/management-of-acute-malnutrition-and-micronutrientdeficiencies-standard-2-severe-acute-malnutrition/

6. Bhutta Z, Das J, Rizvi A, Gaffey MF, Walker N, Horton S, et al. Evidence-based interventions for improvement of maternal and child nutrition: what can be done and at what cost? Maternal and Child Nutrition 2013; 2:1-15.

7. Levels and trends in child malnutrition. UNICEF / WHO / World Bank Group Joint Child Malnutrition Estimates,2017.https://www.who.int/nutgrowthdb/estimates

8. CSA, ICF. Ethiopia Demographic and Health Survey 2017: Key Indicators Report. Addis Ababa, Ethiopia, and Rockville, Maryland, USA 2017.

9. Beyene TT. Predictors of Nutritional Status of Children Visiting Health Facilities in Jimma Zone, South West Ethiopia International Journal of Advanced Nursing Science and Practice

10. WHO. Guideline: Updates on the Management of Severe-Acute Malnutrition in Infants and Children.

$\begin{array}{llll}\text { Geneva: } & \text { world } & \text { health } & \text { organization; }\end{array}$ http://www.who.int/elena/titles/full_recommendations/sam_management/en/2012; 1(1):1-13.

11.Teferi E, Lera M, Sita S, Bogale Z, Datiko DG, Yassin MA. Treatment Outcome of Children with Severe Acute Malnutrition Admitted to Therapeutic Feeding Centers in Southern Region of Ethiopia. Ethiopian Journal of Health Development 2010;24(3):234-38.

12. Saaka M, Osman S, Amponsem A, Ziem JB, Abdul-Mumin A, Akanbong P, et al. Treatment Outcome of Severe Acute Malnutrition Cases at the Tamale Teaching Hospital Journal of Nutrition and Metabolism 2015:18.

13. Kebede S. Survival Status and Predictors of Mortality among Children Aged 0-59 Months with Severe Acute Malnutrition Admitted to Stabilization Center at Sekota Hospital Waghemra Zone. Journal of Nutritional Disorders Therapy 2015; 5(2):160-71.

14. Berti A, Bregani ER, Manenti F, Pizzi C. Outcome of severely malnourished Children treated according to UNICEF 2004 guidelines: a one-year experience in a Zone hospital in rural Ethiopia. Transactions of the royal society of tropical medicine and hygiene 2008;102(9):939-44.

15. Habtamu J, Alemseged W, Fissehaye A. Survival status and predictors of mortality in severely malnourished children admitted to Jimma University Specialized Hospital from 2010 to 2012, Jimma, Ethiopia: a retrospective longitudinal study. BMC pediatrics2015;15(76):1-13. 
16. Gebremichael DY. Predictors of nutritional recovery time and survival status among children with severe acute malnutrition who have been managed in therapeutic feeding centers, Southern Ethiopia: retrospective cohort study. BMC Public Health 2015; 15(1):1-11.

17. Melaku G, Afework MB, Mache T. Treatment Outcomes and Associated Risk Factors of Severely Malnourished under Five Children Admitted to Therapeutic Feeding Centers of Mekelle City, Northern Ethiopia. Open Access Library Journal 2015; 1(4):1-9.

18. Banbeta A, Seyoum D, Belachew T, Birlie B, Getachew Y. Modeling time-to-cure from severe acute malnutrition: application of various parametric frailty models. Archives of Public Health, the official journal of the Belgian Public Health Association, 2015; 73(6):1-8.

19. Maggie P. Critical appraisal of the management of severe acute malnutrition in Malawi: a case of two hospitals in Zomba. 2011; 19-27. http://ummafrapp.de/skandal/haart/annex\%209 .pdf

20. Munthali T, Jacobs C, Sitali L, Dambe R, Michelo c. Mortality and morbidity patterns in under-five children with severe acute malnutrition (SAM) in Zambia: a five-year retrospective review of hospital-based records (2009 -2013). Archives of Public Health 2015; 73(23).

21. Talbert A, Thuo N, Karisa J, Chesaro C, Ohuma E, Ignas J, et al. Diarrhoea Complicating Severe Acute Malnutrition in Kenyan Children: A Prospective Descriptive Study of Risk Factors and Outcome. PLoS ONE 2012; 7(6).

22. Abeje AT, Gudayu TW, Malefia YD, Befftu BB. Analysis of Hospital Records on Treatment Outcome of Severe

Acute Malnutrition: The Case of Gondar University Tertiary Hospital. Pediat Therapeut 2017; 6:283.doi:10.4172/2161-0665.1000283

23. Desyibelew HD, Fekadu A, Woldie H. Recovery rate and associated factors of children age 6 to 59 months admitted with severe acute malnutrition at inpatient unit of Bahir Dar Felege Hiwot Referral hospital therapeutic

feeding unite, northwest Ethiopia. PLoS ONE 2017; 12(2): doi:10.1371/journal.pone.017102.

24. Emotional Stimulation in the Context of Emergency Food Interventions, Play Therapy

Africa: The Society for Protection and Therapeutic Aid in Africa; Final Report, Addis Ababa (2009).

http://www.ennonline.net/emotionalstimulation

25. Nahar B, Hamadani JD, Ahmed T, Tofail F et al., Effects of psychosocial stimulation on Growth and 
development of severely malnourished children in a nutrition unit in Bangladesh.European Journal of Clinical Nutrition (2009) 63,725-731; doi:10.1038/ejcn.2008.44

26. Food and Nutrition Technical Assistance III Project (FANTA).Studying the Use of Antibiotics for Treating Severe Acute Malnutrition. Washington, DC, 2015. https://www.fantaproject.org/.../FANTA-Impact-CMAMAntibiotics-May2015_0.pdf

27. Devan Jaganath and Ezekiel Mupere. Childhood Tuberculosis and Malnutrition. The Journal of Infectious Diseases 2012; 206:1809-15

28. Girum T, Kote M, Tariku B, Bekele H.survival status and predictors of mortality among severely acute malnourished children $<5$ years of age admitted to stabilization centers in gedeo Zone: a retrospective cohort study. Therapeutics and Clinical Risk Management 2017:13 101-110.

29. Chane T, Oljira L, Atomesa GE, Agedew E. Treatment Outcome and Associated Factors among UnderFive Children with Severe Acute Malnutrition Admitted to Therapeutic Feeding Unit in Woldia Hospital, North Ethiopia. J Nutr Food Sci 2015; 4(329) 